\title{
MicroRNAs in type 2 diabetes mellitus: potential role of physical exercise
}

\author{
Alex Cleber Improta-Caria ${ }^{1,2,3, *}$, Ricardo Augusto Leoni De Sousa ${ }^{4}$, \\ Leonardo Roever ${ }^{5}$, Tiago Fernandes ${ }^{6}$, Edilamar Menezes de Oliveira ${ }^{6}$, \\ Roque Aras Júnior ${ }^{1}$, Bruno Solano de Freitas Souza ${ }^{2,7,8}$ \\ ${ }^{1}$ Post-Graduate Program in Medicine and Health, Faculty of Medicine, Federal University of Bahia, 40110-100 Bahia, Brazil \\ ${ }^{2}$ Center for Biotechnology and Cell Therapy, Sao Rafael Hospital, 41253-190 Bahia, Brazil \\ ${ }^{3}$ Department of Physical Education in Cardiology, Brazilian Society of Cardiology, 41170-130 Bahia, Brazil \\ ${ }^{4}$ Physiological Science Multicentric Program, Federal University of Valleys' Jequitinhonha and Mucuri, $39960-000$ Minas Gerais, Brazil \\ ${ }^{5}$ Federal University of Uberlandia, 38400-128 Minas Gerais, Brazil \\ ${ }^{6}$ Laboratory of Biochemistry and Molecular Biology of the Exercise, School of Physical Education and Sport, Sao Paulo University, 05508-030 Sao \\ Paulo, Brazil \\ ${ }^{7}$ Gonçalo Moniz Institute, Oswaldo Cruz Foundation (FIOCRUZ), 40296-710 Bahia, Brazil \\ ${ }^{8}$ D'Or Institute for Research and Education (IDOR), 41253-190 Salvador, Brazil \\ *Correspondence: aleximprotacaria@gmail.com (Alex Cleber Improta-Caria) \\ Academic Editors: Peter Kokkinos and Jonathan Myers \\ Submitted: 30 September 2021 Revised: 19 November 2021 Accepted: 25 November 2021 Published: 17 January 2022
}

\begin{abstract}
Type 2 diabetes mellitus (T2DM) is a multifactorial metabolic disease, and its prevalence has grown worldwide. Several pathophysiological processes contribute to the development, progression and aggravating of the disease, for example, decreased insulin synthesis and secretion, insulin resistance, inflammation, and apoptosis, all these processes are regulated by various epigenetic factors, including microRNAs (miRNAs). MiRNAs are small non-coding RNAs, which are around 20 nucleotides in length and are regulators of gene expression at the post-transcriptional level, have a specific function of inhibiting or degrading a messenger RNA target. Thus, miRNAs modulate the expression of many associated genes with the pathophysiological processes in T2DM. On the other hand, miRNAs are also modulated through physical exercise (PE), which induces a change in their expression pattern during and after exercise. Some scientific evidence shows that PE modulates miRNAs beneficially and improves the signaling pathway of insulin resistance, however, little is known about the function of PE modulating miRNAs associated with the processes of insulin secretion, inflammation, and apoptosis. Thus, the objective of this review is to identify the miRNAs expression pattern in T2DM and compare it with the exercise-induced miRNAs expression pattern, identifying the signaling pathways that these miRNAs are regulating in the processes of insulin secretion, insulin resistance, inflammation, and apoptosis in T2DM, and how PE may have a potential role in modulating these signal transduction pathways, promoting benefits for patients with T2DM.
\end{abstract}

Keywords: Type 2 diabetes; MicroRNAs; Physical exercise

\section{Introduction}

Type 2 diabetes mellitus (T2DM) is a complex metabolic disease that is associated with genetic and environmental factors, such as, genes that predispose defects in insulin synthesis, consumption of hypercaloric foods, physical inactivity, excessive consumption of alcohol and obesity, generating a defect in insulin secretion and signaling [1], leading to hyperglycemia, insulin resistance and hyperinsulinemia [2].

Insulin resistance occurs when target tissues are unable to respond normally to the action of insulin [3]. Under physiological conditions, insulin binds to the insulin receptor, that is a type of tyrosine kinase receptor, starting autophosphorylation of the tyrosine residues, and the addition of phosphate groups generates a binding site for the insulin receptor substrate-1 (IRS-1). The activated IRS-1 initiates the signal transduction pathway activating phosphatidyli- nositol 3-kinase (PI3K) and consequently phosphorylates protein kinase $\mathrm{B}$ (PKB) also called (AKT), which activates the translocation of the glucose transporter 4 (GLUT4) from the cytoplasm to the cell surface [4], however, in insulin resistance, phosphorylation of the serine residue in IRS-1 occurs, decreasing the phosphorylation of PI3K, inhibiting activation of its pathway, impairing glucose uptake [5].

Other molecular mechanisms at the epigenetic level are associated with this defect in insulin signaling and insulin resistance, such as the regulation performed by microRNAs (miRNAs) [6]. MiRNAs are small ribonucleic acids (RNA), do not encode proteins, are around 18 to 25 nucleotides in length, with the function of inhibiting or degrading one or more messenger ribonucleic acids (mRNA) target [7].

Several miRNAs are modulating the insulin signaling pathway and the proteins associated, as well as miR- 
128a, miR-96, miR-126 regulating the expression of the IRS-1; miR-29, miR-384-5p, miR-1 modulating PI3K expression; miR-143, miR-145, miR-29, miR-383, miR-33a, miR-33b, miR-21 regulating AKT expression and miR133a, miR-133b, miR-223, miR-143 modulating the expression of GLUT4 [6], and dysregulation of these miRNAs can result in inhibition of the insulin signaling pathway, insulin resistance and T2DM.

On the other hand, evidence shows that physical exercise (PE) modulates the expression of miRNAs [8-10] and can induce a beneficial modification in signaling pathways in T2DM [11], increasing insulin sensitivity [12], reducing insulin resistance [13], in addition, to being an excellent non-pharmacological strategy to combat T2DM [14]. Thus, the objective of this review is to analyze the expression pattern of miRNAs in T2DM and compare with the expression pattern induced by $\mathrm{PE}$, analyzing the signaling pathways associated with these miRNAs in the pathophysiological processes in T2DM.

\section{Type 2 diabetes mellitus}

The prevalence of T2DM continues to grow worldwide and represents $95 \%$ of all diabetes cases, increasing the incidence of disease-related morbidity and mortality. Genetic factors associated with hypercaloric diet, smoking, excessive alcohol intake and sedentary behavior are essential aspects for the development of insulin resistance and T2DM [15].

Constant hyperglycemia in the body causes increased insulin secretion, leading to chronic hyperinsulinemia associated with hyperglycemia and subsequently beta cell failure in pancreatic islets [16]. All of these factors associated promote impaired insulin signaling, suppressing glucose uptake by cells, decreasing the production of adenosine triphosphate (ATP) by glycolytic pathway, inducing a substrate switch with the predominance of fatty acid metabolism for production of ATP.

Increased lipolysis and elevated free fatty acid levels contributes to further increase glucose output, interfering with insulin signaling. An excess supply of fatty acids causes phosphorylation of the peroxisome proliferatoractivated alpha (PPAR $\alpha$ ), resulting in intramyocardial triglyceride accumulation and increased reactive oxygen species (ROS) generation, which subsequently enhances inflammation, apoptosis, and contractile dysfunction, features characteristic of lipotoxicity [2,17].

$\operatorname{PPAR} \alpha$ has been reported to reduce the mitochondrial uncoupling protein 3 (UCP3) expression in myocytes through activation of the lipogenic factor sterol regulatory element-binding protein (SREBP)-1 and in response to fatty acids, involved with the development of selective insulin resistance in the heart [18]. Accordingly, mice with cardiac specific PPAR $\alpha$ over-expression exhibited a similar phenotype of diabetic cardiomyopathy [19].

Further, study showed krüppel-like factor-5 (KLF5) is as a direct activator of the $\operatorname{PPAR} \alpha$ promoter in mice with streptozotocin-induced type-1 diabetes, indicating it is involved in diabetic cardiomyopathy either via stimulation of PPAR $\alpha$ expression or independently via forkhead box protein $\mathrm{O} 1$ (FOXO1) activation. In agreement, diabetic mice with FOXO1 deletion had lower cardiac KLF5 expression and were protected from diabetic cardiomyopathy [20].

Other transcription factors have been described to regulate glucose uptake. The myocyte enhancer factor 2 (MEF2) appear to regulate the GLUT4 promoter [21]. In fact, MEF2 binding activity was substantially reduced in both heart and skeletal muscle of diabetic mice, which correlated with decreased transcription rate of the GLUT4 gene. These observations have raised the possibility that these transcriptional factors can drive pathophysiology in T2DM.

All of these mechanisms contribute to the progression of insulin resistance and damage to specific cells in T2DM, such as cardiomyocytes, endothelial cells, vascular smooth muscle cells, pancreatic beta cells, among others, generating inflammation, apoptosis, fibroblast activation, fibrosis and cell dysfunction [22], impressively, these mechanisms are regulated by miRNAs at the post-transcriptional level in T2DM [23].

\subsection{MicroRNAs and insulin resistance in T2DM}

Insulin resistance is a pathophysiological process in T2DM in which the target cells do not normally respond to the action of insulin, impairing the signaling of this protein [3]. MiRNAs as regulators of gene expression and consequently, regulators of protein expression [24], modulate the synthesis, secretion of insulin and the expression of proteins in its signaling pathway in T2DM (Table 1, Ref. [25-45]), such as PI3K, AKT and GLUT4 [46].

Evidence shows that miR-375 regulates the process of insulin secretion [47] and glucose homeostasis [48]. MiR96 also participates in this process, reducing the expression of nucleolar complex protein 2 (NOC2) in pancreatic beta cells by decreasing insulin secretion [49]. Other miRNAs that regulate insulin secretion and are associated with the development of pancreatic beta cells are miR-15a [50] and miR-7 [51]. MiR-7 is overexpressed in the serum of T2DM patients [52] and regulates the expression of the IRS-2 gene, reduces the expression of this gene and impairs insulin signaling, inducing insulin resistance [53].

MiR-33a and miR-33b are also highly expressed in T2DM and inhibit the expression of IRS-2, in addition, miR-126 and miR-145 suppress the expression of IRS-1, all of these mechanisms contribute to impact the pathway of insulin signal transduction and promote insulin resistance [54]. Following the insulin signaling pathway, the miRNAs that regulate the PI3K-p85 $\alpha$ subunit are miR-29 [55] and miR-320 [56], while miR-126 controls the expression of p85 $\beta$ subunit [57]. These deregulated miRNAs impact insulin signaling in T2DM. 
Table 1. MiRNAs dysregulated in T2DM (Clinical Studies)

\begin{tabular}{|c|c|c|c|c|}
\hline MicroRNA & Samples & Outcomes & Targets & References \\
\hline$\downarrow$ miR-130a & $\begin{array}{l}\text { Diabetic Patients }(n=72) \\
\text { Peripheral blood samples }\end{array}$ & MiR-130 regulated glucose metabolism & PPARy & $\begin{array}{l}\text { Jiao et al., } 2015 \\
{[30]}\end{array}$ \\
\hline $\begin{array}{l}\uparrow \operatorname{miR}-128, \\
\text { miR-130b-3p, miR-374a-5p }\end{array}$ & $\begin{array}{l}\text { Diabetic Patients }- \text { South Asians } \\
(\mathrm{n}=145) \\
\text { Serum samples }\end{array}$ & $\begin{array}{l}\text { Differentially expressed miRNAs comparing prediabetes and T2DM patients with control } \\
\text { subjects were: miR-128, miR-130b-3p, miR-374a-5p }\end{array}$ & $\begin{array}{l}\text { IRS-1, PI3KR1, SOCS4; insulin } \\
\text { signaling (e.g., insulin receptor, } \\
\text { insulin receptor substrate-1 and } \\
\text { phosphatidylinositol 3-kinases } \\
\text { regulatory 1) }\end{array}$ & $\begin{array}{l}\text { Prabu et al., } 2015 \\
{[31]}\end{array}$ \\
\hline $\begin{array}{l}\uparrow \mathrm{miR}-572 \\
\downarrow \mathrm{miR}-1249, \mathrm{miR}-320 \mathrm{~b}\end{array}$ & $\begin{array}{l}\text { Prediabetic and newly-diagnosed } \\
\text { Diabetic Patients }(\mathrm{n}=150) \\
\text { Plasma samples }\end{array}$ & $\begin{array}{l}\text { MiR-1249, miR-320b, and miR- } 572 \text { in plasma samples were all significantly different among } \\
\text { T2D patients, prediabetes, and controls }\end{array}$ & FOXO1, EIF2AK3 & $\begin{array}{l}\text { Yan et al., } 2016 \\
{[32]}\end{array}$ \\
\hline$\uparrow$ miR-29a, miR-34a, miR-375 & $\begin{array}{l}\text { Prediabetic and newly-diagnosed } \\
\text { Diabetic Patients }(\mathrm{n}=56) \\
\text { Serum samples }\end{array}$ & $\begin{array}{l}\text { Serum analysis of seven miRNAs revealed altered expression levels; miR-34a showed the most } \\
\text { significant differences }\end{array}$ & & $\begin{array}{l}\text { Kong et al., } 2011 \\
{[33]}\end{array}$ \\
\hline$\downarrow$ let-7 & $\begin{array}{l}\text { Diabetic Patients }(\mathrm{n}=4) \\
\text { Carotid endarterectomy specimens } \\
\text { samples } \\
\text { Diabetic mice }(\mathrm{n}=6) \\
\text { Aortic samples }\end{array}$ & Proliferation, migration, inflammation & NF-kB & $\begin{array}{l}\text { Brennan et al., } 2017 \\
{[34]}\end{array}$ \\
\hline$\downarrow \mathrm{miR}-503$ & $\begin{array}{l}\text { Patients with T2DM, obesity or } \\
\text { both ( } \mathrm{n}=56 \text { ) } \\
\text { Serum samples }\end{array}$ & $\begin{array}{l}\text { MiRNAs (miR-138, miR376a and miR-15b) are potential biomarkers to distinguish obesity pat- } \\
\text { ients from obesity-T2D and T2D patients; the combination of miR-503 and miR-138can dis- } \\
\text { tinguish diabetic from obese diabetic patients }\end{array}$ & Insulin & $\begin{array}{l}\text { Pescador } \text { et al., } 2013 \\
{[35]}\end{array}$ \\
\hline $\begin{array}{l}\uparrow \text { miR-140-5p; miR-142-3p; } \\
\text { miR-222 } \\
\downarrow \text { miR-423-5p; miR-125b; miR- } \\
\text { 192; miR-195; miR-130b; miR- } \\
\text { 532-5p; miR-126 }\end{array}$ & $\begin{array}{l}\text { Diabetic Patients }(\mathrm{n}=150) \\
\text { Plasma samples }\end{array}$ & $\begin{array}{l}\text { Levels of four miRNAs (miR-140-5p, miR-423-5p, miR-195, and miR-126) was specific for } \\
\text { T2D }\end{array}$ & $\begin{array}{l}\text { INS, IRS-1 } \\
\text { PI3KR2, CXCL2, NFKBIZ }\end{array}$ & $\begin{array}{l}\text { Ortega et al., } 2014 \\
\text { [29] }\end{array}$ \\
\hline$\uparrow \operatorname{miR}-144$ & $\begin{array}{l}\text { Diabetic Patients }(n=50) \\
\text { Serum samples }\end{array}$ & $\begin{array}{l}\text { miR-144 expression was upregulated in whole blood of T2D patients, concordant with the down- } \\
\text { regulation of IRS1;miR-144 exhibited a relationship with increasing glycaemic status }\end{array}$ & IRS-1 & $\begin{array}{l}\text { Karolina et al., } 2011 \\
\text { [26] }\end{array}$ \\
\hline $\begin{array}{l}\uparrow \text { miR-455-5p; miR-454-3p; } \\
\text { miR-144-3p; miR-96-5p } \\
\downarrow \text { miR-409-3p; miR-665; miR- } \\
766-3 p\end{array}$ & Diabetic Patients ( $\mathrm{n}=15)$ & $\begin{array}{l}\text { The expression levels of miR-455-5p, miR-454-3p, miR-144-3p and miR-96-5p were higher in pat- } \\
\text { ients with T2DM, compared with those of healthy subjects, however, the levels of miR-409-3p, miR- } \\
665 \text { and miR-766-3p were lower }\end{array}$ & $\begin{array}{l}\text { SOCS3, RPS6KB1; TNF, TN- } \\
\text { FRSF1B, PRKAA2, SLC2A1 } \\
\text { PRKAA1; PPARA, PDE3B, FO- } \\
\text { XO1, MAP2K; AKT1, IRS1, IRS2 }\end{array}$ & Yang et al., 2017 [28] \\
\hline
\end{tabular}


Table 1. Continued.

\begin{tabular}{|c|c|c|c|c|}
\hline MicroRNA & Samples & Outcomes & Targets & References \\
\hline$\uparrow \operatorname{miR}-101$, miR-375, miR-802 & $\begin{array}{l}\text { Diabetic Patients }(\mathrm{n}=204) \\
\text { Serum samples }\end{array}$ & MiR-101, miR-375 and miR-802 are significantly increased in T2D patients versus NGT subjects & INS, Mtpn, EZH2, Hnflb & $\begin{array}{l}\text { Higuchi et al., } 2015 \\
{[36]}\end{array}$ \\
\hline $\begin{array}{l}\downarrow \text { miR-23a, miR-186, miR-191, } \\
\text { miR-146a, let-7i }\end{array}$ & $\begin{array}{l}\text { Diabetic Patients }(n=60) \\
\text { Serum samples }\end{array}$ & Revealed low levels of miR-23a, let-7i, miR-486, miR-186, miR-191, miR-192, and miR-146a in T2D & $\mathrm{NF}-\mathrm{kB}$ & Yang et al., 2014 [37] \\
\hline$\uparrow \mathrm{miR}-1, \mathrm{miR}-133 \mathrm{a}$ & $\begin{array}{l}\text { Diabetic Patients }(n=86) \\
\text { Serum samples }\end{array}$ & $\begin{array}{l}\text { MiR-133a levels were increased in type } 2 \text { diabetes patients as compared with healthy subjects; miR-1 } \\
\text { and miR-133a levels were robustly associated with myocardial steatosis in type } 2 \text { diabetes patients }\end{array}$ & MEF2a & $\begin{array}{l}\text { Gonzalo-Calvo et al., } \\
2017 \text { [38] }\end{array}$ \\
\hline$\downarrow$ miR-126 & $\begin{array}{l}\text { Diabetic Patients }(\mathrm{n}=822) \\
\text { Plasma samples }\end{array}$ & $\begin{array}{l}\text { Lower levels of miR-20b, miR-21, miR-24, miR-15a, miR-126, miR-191, miR-197, miR-223, miR-320, and } \\
\text { miR-486 in prevalent DM, but a modest increase of miR-28-3p }\end{array}$ & PI3KR2, SPRAD1 & $\begin{array}{l}\text { Zampetaki et al., } \\
2010[39]\end{array}$ \\
\hline$\downarrow$ miR-126 & $\begin{array}{l}\text { Diabetic Patients }(n=40) \\
\text { Plasma samples }\end{array}$ & $\begin{array}{l}\text { MiR-126 expression was decreased before the manifestation of T2DM. MiR-126 level was inversely ass- } \\
\text { ociated with the onset of DM }\end{array}$ & PI3KR2, SPRAD1 & Zhang et al., 2015 [40] \\
\hline$\downarrow$ miR-126 & $\begin{array}{l}\text { Diabetic Patients }(\mathrm{n}=455) \\
\text { Serum samples }\end{array}$ & MiR-126 was reduced in T2DM patients. & PI3KR2, SPRAD1 & Liu et al., 2014 [41] \\
\hline$\downarrow$ miR-126 & $\begin{array}{l}\text { Diabetic Patients }(\mathrm{n}=300) \\
\text { Plasma samples }\end{array}$ & $\begin{array}{l}\text { MiR-126-3p and miR-21-5p levels declined significantly from CTR to T2DM non complicated and T2DM } \\
\text { complicated patients }\end{array}$ & PI3KR2, SPRAD1 & Olivieri et al., 2015 [42] \\
\hline $\begin{array}{l}\uparrow \text { miR-30a-5p, miR-150 } \\
\downarrow \text { miR-15a, miR-375 }\end{array}$ & $\begin{array}{l}\text { Diabetic Patients }(\mathrm{n}=462) \\
\text { Plasma samples }\end{array}$ & $\begin{array}{l}\text { It was identified that miRNAs are dysregulated before the development of type } 2 \text { diabetes and confirmed the ex- } \\
\text { pression profile after the disease is established in individuals, demonstrating the potential predictor of these genes }\end{array}$ & & $\begin{array}{l}\text { Jiménez-Lucena et al., } \\
2018 \text { [27] }\end{array}$ \\
\hline$\uparrow \operatorname{miR}-424$ & $\begin{array}{l}\text { Diabetic Patients }(\mathrm{n}=122) \\
\text { Serum samples }\end{array}$ & $\begin{array}{l}\text { MiR- } 424 \text { expression increased after Actinidia chinensis juice consumption promoting anti-inflammatory and anti- } \\
\text { oxidative effects }\end{array}$ & KEAP1, NRF2 & Sun et al., 2017 [43] \\
\hline$\downarrow \mathrm{miR}-146 \mathrm{a}$ & $\begin{array}{l}\text { Diabetic Patients }(\mathrm{n}=56) \\
\text { Serum samples }\end{array}$ & $\begin{array}{l}\text { Reduction of miR-146a expression is associated with a proinflammatory state and increased expression of cytokines } \\
\text { such as IL-8, TNF- } \alpha \text {, IL-6 and IL-1 } \beta\end{array}$ & & Baldeón et al., 2014 [25] \\
\hline $\begin{array}{l}\uparrow \mathrm{miR}-21, \mathrm{miR}-24, \mathrm{miR}- \\
34 \mathrm{a}, \mathrm{miR}-148\end{array}$ & $\begin{array}{l}\text { Diabetic Patients }(\mathrm{n}=58) \\
\text { Blood samples }\end{array}$ & $\begin{array}{l}\text { MicroRNAs associated with pancreatic islet } \beta \text { cell function and glycemic control, as well as the development and } \\
\text { progression of type } 2 \text { diabetes }\end{array}$ & SFRP4 & $\begin{array}{l}\text { Nunez Lopez et al., } 2016 \\
\text { [44] }\end{array}$ \\
\hline $\begin{array}{l}\uparrow \mathrm{miR}-21, \mathrm{miR}-30 \mathrm{~d}, \mathrm{miR}- \\
34 \mathrm{a}, \mathrm{miR}-148 \mathrm{a}\end{array}$ & $\begin{array}{l}\text { Diabetic Patients }(\mathrm{n}=92) \\
\text { Plasma samples }\end{array}$ & $\begin{array}{l}\text { MicroRNAs associated with insulin resistance, pancreatic islet } \beta \text { cell function and glycemic control, inflammation, } \\
\text { apoptosis, as well as progression of type } 2 \text { diabetes }\end{array}$ & Insulin & Seyhan et al., 2016 [45] \\
\hline
\end{tabular}


MiRNAs such as miR-29a, miR-29b, miR-29c [58], miR-33a, miR-33b [59] and miR-153 [60] act by inhibiting AKT phosphorylation. Finishing the insulin signaling cascade, GLUT4 is regulated by several miRNAs, such as miR-21a, miR-29a, miR-29c, miR-93, miR-106b, miR133a, miR-133b, miR-222, and miR-223, these inhibit GLUT4 expression and contribute to insulin resistance process in T2DM [61], and this process favors inflammation in various organs and systems in patients with T2DM, who are also mediated by miRNAs.

\subsection{MicroRNAs and inflammation in T2DM}

The inflammatory process is a very important within the pathophysiology of T2DM that affects organs and systems, miRNAs also regulate this process. Some miRNAs are associated with increased pro-inflammatory, such as miR-146a which is downregulated in the serum of T2DM patients [25]. Conversely, the miR-147 has been identified overexpressed in the serum of diabetic and obese rats with periodontitis, activating macrophages and increasing the expression of pro-inflammatory markers such as tumor necrosis factor- $\alpha$ (TNF- $\alpha$ ), nitric oxide synthase-2 (NOS2) and interleukin-12 (IL-12) [62].

The increased levels of TNF- $\alpha$, for example, leads to peripheral inflammation that affects central nervous system functioning and plays an important role in memory loss and cognitive decline, which are seen in T2DM and Alzheimer's disease [63], including, various miRNAs regulate inflammatory processes in both T2DM [11] and Alzheimer's disease [64].

Another miRNA associated to the inflammatory process in T2DM is miR-29c, which is overexpressed by regulating the tristetraprolin (TTP) gene. TTP is recognized for its anti-inflammatory function, but when serum and urinary levels of this protein are decreased an inflammatory response increases the expression of interleukin-6 (IL-6) and interleukin-18 (IL-18) in diabetic nephropathy by a miR29c-regulated molecular mechanism that induces systemic and tissue inflammation in T2DM [65].

A recent meta-analysis revealed that increasing IL6 levels in the blood might be associated with cognitive decline [66] an increase prevalence of dementia and Alzheimer's disease [67]. Inflammation and insulin resistance that happens in T2DM affects not only periphery, but also central nervous system and a link between T2DM and the increased risk of developing neurological disorders, such as Alzheimer's disease, has been reported [68].

Several evidences show that PE is an important nonpharmacological tool for the prevention and treatment of T2DM [69], improving the function of cells of the innate and adaptive immune system [70], reducing inflammation in T2DM [71], including, various training protocols are beneficial for individuals with Alzheimer's disease [72]. Both, aerobic or resistance $\mathrm{PE}$ can reduce insulin resistance and inflammatory profile in individuals with T2DM [73] or
Alzheimer's disease, potentially modulating the expression of miRNAs and signaling pathways [64].

\section{Type 2 diabetes mellitus and physical exercise}

T2DM comprises up to $95 \%$ of diabetes cases worldwide [74]. PE can prevent and fight insulin resistance that occurs in T2DM [75]. PE protocols include mainly aerobic and resistance sessions and/or training, which should be prescribed based on scientific variables, such as volume, frequency, intensity, and duration [76].

Aerobic PE protocols lasting 30-60 minutes, 5 days/week revealed to enhance insulin sensitivity and cardiovascular functioning, reducing the risk for developing T2DM [77]. Resistance training protocols have also showed to reduce hyperglycemia in T2DM [78].

In general, PE training enhances peroxisome proliferator-activated receptor gamma coactivator-1 alpha (PGC-1 $\alpha$ ) levels inducing the remodeling of skeletal muscle fiber composition, by leading to the browning of the adipose tissue and facilitating conversion of muscle fibers from oxidative to glycolytic and vice-versa, depending on if it is adopted an aerobic or resistance training, respectively [79]. PE leads to the production of irisin through the PGC-1 $\alpha$ pathway having a role in T2DM [75].

Irisin is a myokine identified for its ability to induce the physiological changes contribute to prevent and avoid the development of insulin resistance and inflammation in T2DM [80]. Irisin is an important mediator of the beneficial effects of PE on metabolic and neurological profiles $[81,82]$. Thus, PE is an important non-pharmacological approach to reduce the risk of developing or worsening T2DM pathology.

\subsection{MicroRNAs in T2DM and physical exercise}

Homeostasis at the cellular level is affected by a single session of PE and also in response to chronic exercise inducing alterations in the circulating and cellular miRNA profile (Table 2, Ref. [83-109]) [11]. In both healthy and diabetic individuals, different types of PE modulate the expression of miRNAs $[83,110]$. Resistance and aerobic training promote changes in the expression of circulating microRNAs, specifically, miR-423-3p, miR-451a and miR-766-3p were upregulated in the plasma of diabetic patients after both training, and changes in miR-423-3p and miR-451a were correlated with body fat loss and with changes in fatty acid metabolism [111].

Aerobic exercise (cycling or exercising on an elliptical crosstrainer) induced a decrease in the expression of miR$29 b-3 p$, miR-29c-3p and miR-135a-5p post-training in the skeletal muscle of diabetic individuals, including the reduction of miR-29c-3p was correlated with improvement in cardiorespiratory fitness [112]. Swimming training, another type of aerobic exercise, also induced a change in the expression of another miRNA, miR-382 was overexpressed 
Table 2. MiRNAs in PE (Clinical Studies). PE modulating miRNAs in both the acute and chronic phases.

\begin{tabular}{|c|c|c|c|c|}
\hline MicroRNAs & Targets & Source & Exercise Protocols & Reference \\
\hline $\begin{array}{l}\uparrow \text { miR-125a, miR-145, miR-181b, miR-193a, miR-197, miR-212, miR-223, } \\
\text { miR-340, miR-365, miR-485, miR-505, miR-520d, miR-629, miR-638, mi- } \\
\text { R-939, miR-940, miR-1225, miR-1238 }\end{array}$ & & Healthy men $(n=11)$ & Acute Response & Radom-Aizik et al., 2010 [85] \\
\hline $\begin{array}{l}\downarrow \text { miR-let-7i, miR-16, miR-17, miR-18a, miR-18b, miR-20a, miR-20b, miR- } \\
\text { 22, miR-93, miR-96, miR-106a, miR-107, miR-126, miR-130a, miR-130b, } \\
\text { miR-151, miR-185, miR-194, miR-363, miR-660 }\end{array}$ & & Serum samples & $\begin{array}{l}\text { Cycle ergometer exercise }(10 \times 2 \text { min bouts, } 1 \text { min rest interval between } \\
\text { each bout, } 76 \% \text { VO2peak) }\end{array}$ & \\
\hline$\uparrow$ miR-21, miR-146a, miR-221, miR-222, miR-20a & $\begin{array}{l}\text { PTEN } \\
\text { PDCD4 } \\
\text { p27/KIP1 } \\
\text { p21/WAF 1 }\end{array}$ & $\begin{array}{l}\text { Healthy men }(\mathrm{n}=10) \\
\text { Plasma samples }\end{array}$ & $\begin{array}{l}\text { Acute Response } \\
\text { Cardiopulmonary exercise test } \\
\text { Chronic Adaptation ( } 90 \text { days) } \\
\text { Rowing training, } 5 \mathrm{Km}, 1-3 \mathrm{~h} \text { per session, } 20-24 \text { strokes } / \text { min }\end{array}$ & Baggish et al., 2011 [88] \\
\hline $\begin{array}{l}\uparrow \text { miR-7, miR-15a, miR-21, miR-26b, miR-132, miR-140, miR-181a, miR- } \\
\text { 181b, miR-181c, miR-338, miR-363, miR-939, miR-940, miR-1225 } \\
\downarrow \text { let-7e, miR-23b, miR-31, miR-99a, miR-125a, miR-125b, miR-126, miR- } \\
\text { 130a, miR-145, miR-151, miR-199a, miR-199b, miR-221, miR-320, miR- } \\
\text { 451, miR-486, miR-584, miR-652 }\end{array}$ & & Healthy men $(n=12)$ & $\begin{array}{l}\text { Acute Response } \\
\text { Cycle ergometer exercise }(10 \times 2 \text { min bouts, } 1 \text { min rest interval between } \\
\text { each bout, } 76 \% \text { VO2peak) }\end{array}$ & Radom-Aizik et al., 2012 [84] \\
\hline $\begin{array}{l}\uparrow \mathrm{miR}-149 \\
\downarrow \mathrm{miR}-146 \mathrm{a}, \mathrm{miR}-221\end{array}$ & & $\begin{array}{l}\text { Healthy men }(n=12) \\
\text { Serum samples }\end{array}$ & $\begin{array}{l}\text { Acute Response } \\
\text { Resistance exercise (bench press and leg press) } 3 \text { days after exercise }\end{array}$ & Sawada et al., 2013 [89] \\
\hline$\downarrow \mathrm{miR}-486$ & PTEN & $\begin{array}{l}\text { Healthy men }(\mathrm{n}=11) \\
\text { Serum samples }\end{array}$ & $\begin{array}{l}\text { Acute Response } \\
\text { Cycle ergometry } 60 \mathrm{~min} \text { at } 70 \% \mathrm{VO} 2 \mathrm{max} \\
\text { Chronic Adaptation (4 weeks total) } \\
\text { Systematic — cycling at } 70 \% \text { VO2max }(3 \times 30 \mathrm{~min} / \text { week })\end{array}$ & Aoi et al., 2013 [90] \\
\hline $\begin{array}{l}\uparrow \text { miR-7, miR-29a, miR-29b, miR-29c, miR-30e, miR-142, miR-192, miR- } \\
\text { 338, miR-363, miR-590 } \\
\downarrow \text { let-7e, miR-126, miR-130a, miR-151, miR-199a, miR-221, miR-223, miR- } \\
\text { 326, miR-328, miR-652 }\end{array}$ & & Healthy men $(n=13)$ & $\begin{array}{l}\text { Acute Response } \\
\text { Cycle ergometer exercise }(10 \times 2 \text { min bouts, } 1 \text { min rest interval between } \\
\text { each bout, } 77 \% \text { VO2peak })\end{array}$ & Radom-Aizik et al., 2013 [86] \\
\hline$\uparrow$ miR-181b, miR-214, miR-1, miR-133a, miR-133b, miR-208b & & $\begin{array}{l}\text { Healthy men }(\mathrm{n}=9) \\
\text { Plasma samples }\end{array}$ & $\begin{array}{l}\text { Acute Response } \\
\text { Uphill treadmill test (concentric) } \\
\text { Immediately after Downhill treadmill test (eccentric) } 2-6 \mathrm{~h} \text { after exercise }\end{array}$ & Banzet et al., 2013 [91] \\
\hline$\uparrow \operatorname{miR}-126, \mathrm{miR}-133$ & $\mathrm{CPK}$ & $\begin{array}{l}\text { Healthy men }(\mathrm{n}=58) \\
\text { Plasma samples }\end{array}$ & $\begin{array}{l}\text { Acute Response } \\
\text { Single symptom-limited spiroergometry test } \\
\text { Cycling } 4 \mathrm{~h} \text { at } 70 \% \text { of anaerobic threshold } \\
\text { Marathon run } \\
\text { Eccentric resistance exercise }\end{array}$ & Uhlemann et al., 2014 [92] \\
\hline
\end{tabular}


Table 2. Continued.

\begin{tabular}{|c|c|c|c|c|}
\hline MicroRNAs & Targets & Source & Exercise Protocols & Reference \\
\hline \multirow{3}{*}{$\uparrow$ miR-1, miR-126, miR-133a, miR-134, miR-146a, miR-208a, miR-499 } & CPK & Healthy men $(n=21)$ & Acute Response & \multirow{3}{*}{ Baggish et al., 2014 [93] } \\
\hline & NT-proBNP & & Marathon run & \\
\hline & hsCRP & Plasma samples & Immediately after run (decreased after $24 \mathrm{~h}$ ) & \\
\hline \multirow[t]{3}{*}{$\uparrow$ miR-1, miR-133a, miR-206, miR-208b, miR-499 } & & Healthy men $(n=14)$ & Acute Response & \multirow{3}{*}{ Mooren et al., 2014 [94] } \\
\hline & & \multirow{2}{*}{ Plasma samples } & Marathon run & \\
\hline & & & Immediately after run & \\
\hline \multirow{3}{*}{$\uparrow$ miR-1, miR-133a, miR-206 } & & Healthy men $(n=5)$ & Acute Response & \multirow{3}{*}{ Gomes et al., 2014 [95] } \\
\hline & & \multirow{2}{*}{ Plasma samples } & Marathon run & \\
\hline & & & Immediately after run & \\
\hline $\begin{array}{l}\uparrow \text { miR-15a, miR-29b, miR-29c, miR-30e, miR-140, miR-324, miR-338, miR- } \\
\text { 362, miR-532, miR-660 }\end{array}$ & & Healthy men $(\mathrm{n}=12)$ & Acute Response & \multirow{2}{*}{$\begin{array}{l}\text { Radom-Aizik et al., } 2014 \\
\text { [87] }\end{array}$} \\
\hline$\downarrow$ miR-23b, miR-130a, miR-151, miR-199a, miR-221 & & Serum samples & $\begin{array}{l}\text { Cycle ergometer exercise }(10 \times 2 \text { min bouts, } 1 \text { min rest interval between } \\
\text { each bout, } 82 \% \text { VO2max })\end{array}$ & \\
\hline \multirow{2}{*}{$\begin{array}{l}\uparrow \text { miR-1, miR-133a, miR-133b, miR-139-5p, miR-143, miR-145, miR-223, } \\
\text { miR-330-3p, miR-338-3p, miR-424 } \\
\downarrow \text { miR-30b, miR-106a, miR-146, miR-151-3p, miR-151-5p, miR-221, miR- } \\
\text { 652, let-7i }\end{array}$} & & \multirow[t]{2}{*}{ Healthy men $(\mathrm{n}=32)$} & Acute Response cycle ergometry test at $65 \%$ Pmax $1-3 \mathrm{~h}$ after exercise & \multirow{4}{*}{ Nielsen et al., 2014 [83] } \\
\hline & & & Immediately after exercise & \\
\hline$\uparrow \operatorname{miR}-103, \operatorname{miR}-107$ & & \multirow{2}{*}{ Plasma samples } & Adaptation (12 weeks total) & \\
\hline $\begin{array}{l}\downarrow \text { miR-21, miR-25, miR-29b, miR-92a, miR-133a, miR-148a, miR-148b, miR- } \\
\text { 185, miR-342-3p, miR-766, let-7d }\end{array}$ & & & Systematic endurance cycle ergometry training, $3-5$ days after training & \\
\hline \multirow[t]{2}{*}{$\uparrow$ miR-1, miR-133a, miR-133b, miR-206, miR-208b, miR-499 } & & Healthy adults $(\mathrm{n}=7)$ & Adaptation (5 months total) & \multirow{2}{*}{ Zhang et al., 2015 [96] } \\
\hline & & Plasma samples & Systematic resistance training $36-72 \mathrm{~h}$ after training & \\
\hline \multirow{2}{*}{$\begin{array}{l}\uparrow \text { let-7f, miR-21, miR-29c, miR-223 } \\
\downarrow \text { let-7f, miR-21, miR-29c, miR-223 }\end{array}$} & & Healthy adults $(n=13)$ & Adaptation (18 weeks) & \multirow{2}{*}{ Dias et al., 2015 [97] } \\
\hline & & PBMCs samples & Running exercise ( $3 \times /$ week, $60 \mathrm{~min})$ & \\
\hline \multirow{3}{*}{$\uparrow \operatorname{miR}-222$} & \multirow{3}{*}{ HIPK1 } & Heart failure patients $(n=28)$ & Acute Response & \multirow{3}{*}{ Liu et al., 2015 [98] } \\
\hline & & Blood samples & Heart failure patients & \\
\hline & & Blood samples & Bicycle Ergometry Test & \\
\hline \multirow[t]{2}{*}{$\begin{array}{l}\uparrow \text { let-7d-3p, let-7f-3p, miR-29a-3p, miR-34a-5p, miR-125b-5p,miR-132-3p, } \\
\text { miR-143-3p, miR-148a-3p, miR-223-3p, miR-223-5p,miR-424-3p, miR-424-5p }\end{array}$} & & Healthy men $(n=9)$ & Acute Response & \multirow{2}{*}{$\begin{array}{l}\text { De Gonzalo-Calvo et al., } \\
2015 \text { [99] }\end{array}$} \\
\hline & & Serum samples & $\begin{array}{l}\text { Marathon run } \\
\text { Immediately after run (decreased after } 24 \mathrm{~h} \text { ) }\end{array}$ & \\
\hline
\end{tabular}


Table 2. Continued.

\begin{tabular}{|c|c|c|c|c|}
\hline MicroRNAs & Targets & Source & Exercise Protocols & Reference \\
\hline \multirow[t]{2}{*}{$\uparrow$ miR-1, miR-30a, miR-133a } & & \multirow{2}{*}{ Healthy adults $(\mathrm{n}=30)$} & Acute Response & \multirow{4}{*}{ Clauss et al., 2016 [100] } \\
\hline & & & Marathon run & \\
\hline \multirow{2}{*}{$\downarrow$ miR-26a, miR-29b } & & \multirow{2}{*}{ Plasma samples } & Immediately after run (decreased after $24 \mathrm{~h}$ ) & \\
\hline & & & Immediately after run & \\
\hline \multirow{3}{*}{$\uparrow$ miR-1, miR-133a, miR-206 } & & Statin and nonstatin-using & Acute Response & \multirow{3}{*}{ Min et al., 2016 [101] } \\
\hline & & runners $(\mathrm{n}=56)$ & Marathon run & \\
\hline & & Plasma samples & Immediately after run (decreased after $24 \mathrm{~h}$ ) & \\
\hline \multirow{4}{*}{$\begin{array}{l}\uparrow \text { miR-1, miR-133a, miR-133b, miR-206, miR-485-5p, miR-509-5p, miR-517a, } \\
\text { miR-518f, miR-520f, miR-522, miR-553, miR-888 }\end{array}$} & \multirow{4}{*}{$\mathrm{NF}-\kappa \mathrm{B}$} & \multirow[b]{2}{*}{ Healthy men $(n=26)$} & Acute Response & \multirow{4}{*}{ Cui et al., 2016 [102] } \\
\hline & & & $\begin{array}{l}\text { High intensity interval exercise } 85-95 \% \text { of HRmax } \\
\text { Immediately after }\end{array}$ & \\
\hline & & \multirow[t]{2}{*}{ Plasma samples } & Vigorous intensity continuous exercise & \\
\hline & & & Immediately after & \\
\hline \multirow[t]{2}{*}{$\uparrow \operatorname{miR}-1$, miR-486, miR-494 } & HDAC4 & Healthy men $(\mathrm{n}=128)$ & Acute Response & \multirow[b]{2}{*}{ Denham et al., 2016 [103] } \\
\hline & $\begin{array}{l}\text { PTEN } \\
\text { FOXO1A }\end{array}$ & Blood samples & $\begin{array}{l}\text { Aerobic exercise VO2max test (Endurance athletes, runners, cyclists and } \\
\text { triathletes) }\end{array}$ & \\
\hline$\uparrow \operatorname{miR}-376 \mathrm{a}$ & & Obese older adults $(\mathrm{n}=33)$ & Adaptation ( 5 months total) & \multirow{2}{*}{ Zhang et al., 2017 [104] } \\
\hline$\downarrow$ miR-16, miR-27a, miR-28 & & Plasma samples & Aerobic run exercise training $30 \mathrm{~min}, 65-70 \%$ heart rate res. (4 days/week) & \\
\hline$\uparrow \operatorname{miR}-21, \operatorname{miR}-16$, miR-93, miR-222 & & Healthy men $(n=30)$ & Adaptation & \multirow{2}{*}{ Horak et al., 2018 [105] } \\
\hline$\downarrow$ miR-222, miR-16 & & Plasma samples & $\begin{array}{l}8 \text { weeks of: Explosive strength training, Hypertrophic strength training, } \\
\text { High-intensity interval training }\end{array}$ & \\
\hline \multirow{2}{*}{$\begin{array}{l}\uparrow \text { miR-221 } \\
\downarrow \text { miR-208b, miR-221, miR-21, miR-146a, miR-210 }\end{array}$} & & Healthy men $(n=10)$ & Acute Response and Adaptation & \multirow{2}{*}{ Li et al., 2018 [106] } \\
\hline & & Serum samples & Basketball Exercise (3-months) & \\
\hline $\begin{array}{l}\uparrow \text { miR-21-5p, miR-27a-3p, miR-29a-3p, miR-30a-5p, miR-34a-5p, miR-126- } \\
\text { 3p, miR-132-3p, miR-142-5p, miR-143-3p, miR-150-5p, miR-195-5p, miR- } \\
\text { 199a-3p }\end{array}$ & & Healthy men $(n=9)$ & Acute Response & \multirow[t]{2}{*}{$\begin{array}{l}\text { De Gonzalo-Calvo et al., } \\
2018 \text { [107] }\end{array}$} \\
\hline $\begin{array}{l}\downarrow \text { miR-16-5p, miR-29b-3p, miR-30b-5p, miR-103a-3p, miR-106b-5p, miR- } \\
\text { 107, miR-139-3p, miR-375, miR-497-5p, miR-590-5p }\end{array}$ & & Serum samples & $10 \mathrm{Km}$ race, half-marathon, marathon & \\
\hline \multirow[t]{2}{*}{$\downarrow$ miR-21-5p, miR-150-5p } & & $\begin{array}{l}\text { Myasthenia Gravis patients } \\
(\mathrm{n}=10)\end{array}$ & Adaptation & \multirow[t]{2}{*}{ Westerberg et al., $2017[108$} \\
\hline & & Serum samples & Aerobic and resistance training twice weekly for 12 weeks & \\
\hline \multirow{2}{*}{$\downarrow$ miR-1-3p, miR-26a-5p, miR-29a-3p, miR-133b, miR-206, and miR-378-5p } & & Healthy men $(\mathrm{n}=17)$ & Acute Response & \multirow{2}{*}{ Margolis et al., 2018 [109] } \\
\hline & & Skeletal muscle samples & Treadmill exercise, $40 \%$ VO2pico & \\
\hline
\end{tabular}




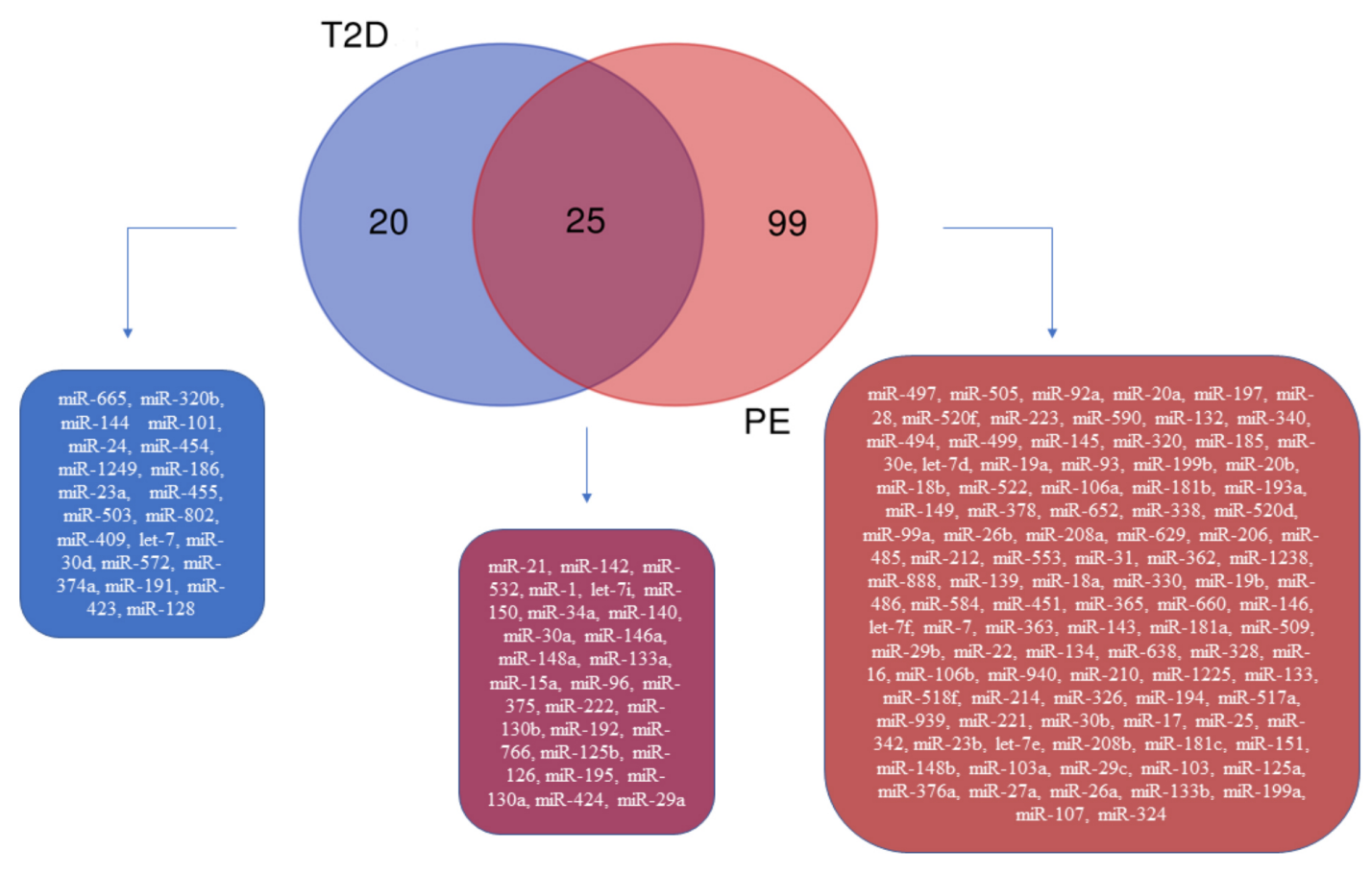

Fig. 1. Overlapping between miRNAs in T2DM and PE. In this Venn Diagram, deregulated miRNAs in T2DM (in blue), miRNAs modulated by PE (in pink) and miRNAs that were modulated in both T2DM and PE (in purple) were identified.

in the serum of diabetic mice after 12 weeks of training. The elevation of miR-382 reduced the expression of the resistin gene, attenuating insulin resistance in these animals [13].

In another study, however with Wistar rats, aerobic training on a treadmill generated overexpression of miR$27 \mathrm{a}$ and miR-27b in cardiac tissue after ten weeks of training [113]. On the other hand, it has already been shown that miR-27a and miR-27b are downregulated during adipocyte differentiation and synthesis [114]. It may be a molecular mechanism by which PE attenuates the differentiation into adipocytes, favoring the reduction of adipose tissue and improvement of insulin resistance.

The enhancement in the expression of miR-27a and miR-27b is probably involved in the control of body mass [115]. Thus, the production of irisin by PE practice may be possibly influencing the regulation of these miRNAs and vice-versa. There are yet many unknown mechanisms linking physiological changes to miRNAs up or down regulation, such as irisin release and its relation to miR-27a and miR-27b. Elucidating these linking mechanisms can contribute significantly to the development of new therapies towards T2DM.

Furthermore, some miRNAs are overexpressed in T2DM and after chronic PE they have the opposite expression. For example, miR-144 is overexpressed in T2DM regulating IRS- 1 and IRS- 2 expression in PI3K pathway [26]. The disturbance of IRS- 1 and IRS-2 functioning is the main molecular mechanism in PI3K pathway that leads to insulin resistance in T2DM [75]. Conversely, it has been reported down-regulation of miR-144 when under PE stress
[113]. There are also data that some miRNAs are downregulated in T2DM whereas after PE these same miRNAs are up-regulated.

Different PE protocols should test different approaches on the volume, frequency, intensity, and duration of the training to evaluate what would be the miRNAs behavior. It is important to emphasize that different behaviors in the expression of miRNAs vary not only with the different PE protocols used, but also with the type of tissue analyzed. Elucidating all of these factors in the future, may guide researchers in choosing the best PE protocols to be used in patients with T2DM.

\subsection{Overlapping between microRNA profile in T2DM and physical exercise}

For a better understanding of the impact of PE on microRNAs in T2DM, a Venn diagram was made (Fig. 1). Of all miRNAs analyzed, 20 miRNAs were identified only in T2DM and 99 miRNAs were regulated by PE alone.

Interestingly, 25 miRNAs have been identified in both T2DM and PE, they are: miR-21, miR-142, miR-532, miR1, let-7i, miR-150, miR-34a, miR-140, miR-30a, miR146a, miR-148a, miR-133a, miR-15a, miR-96, miR-375, miR-222, miR-130b, miR-192, miR-766, miR-125b, miR126, miR-195, miR-130a, miR-424 and miR-29a. Of these 25 miRNAs, we identified 4 miRNAs with different expression pattern in T2DM compared to PE, they are: miR-15a, miR-96, miR-192, miR-532 (Fig. 2), which may be potential beneficial molecular mechanisms regulated by $\mathrm{PE}$ in T2DM. 


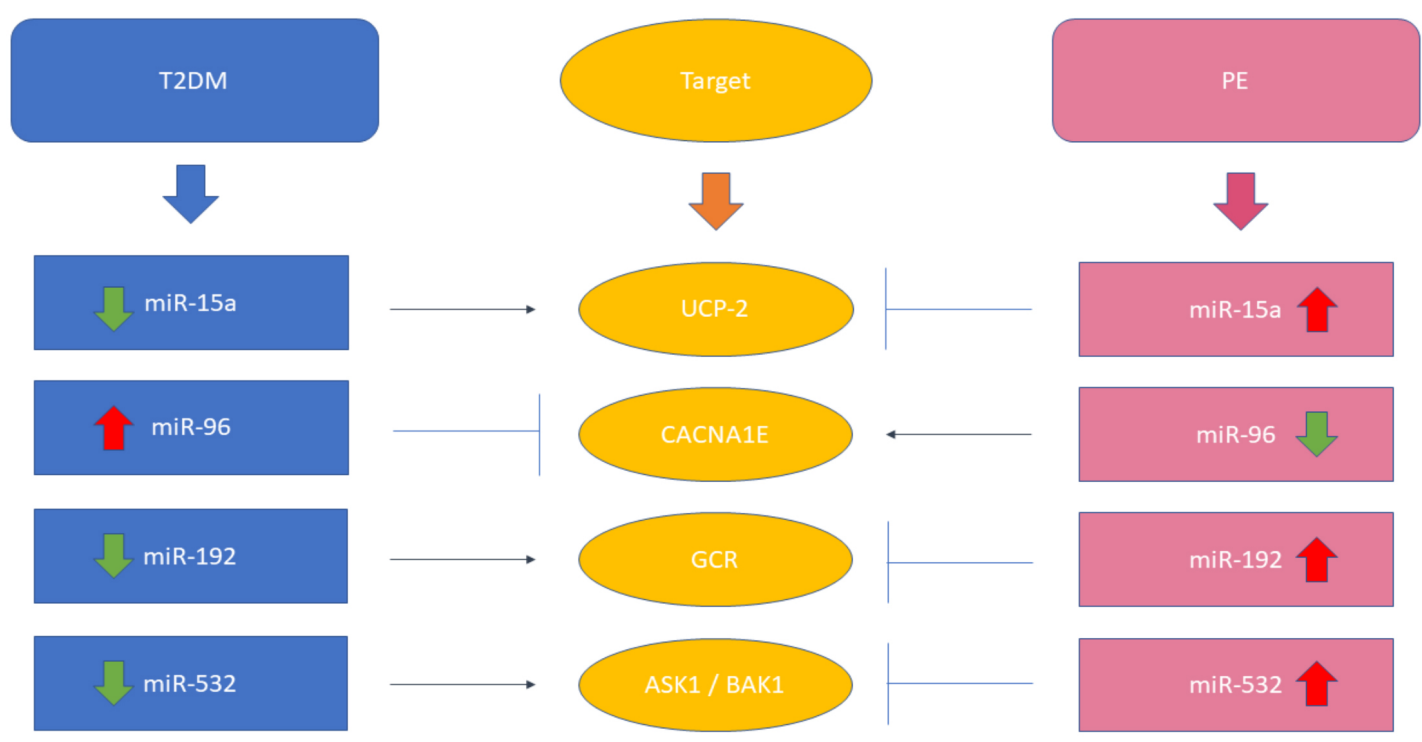

Fig. 2. MiRNAs with different expression patterns between T2DM and PE regulating specific genes. MiRNAs with modified expression in T2DM (in blue), which had opposite expression induced by PE (in pink), regulating target genes (in yellow).

MiR-15a expression is reduced in the plasma of individuals with T2DM and is associated with the development of the disease [27]. MiR-15a regulates the uncoupling protein-2 (UCP-2) gene that impairs insulin synthesis and secretion [116]. Thus, with reduced miR-15a expression and increased UCP-2 in beta cells, insulin secretion is impaired [117], favoring insulin resistance. In contrast, PE on an ergometer cycle induced increased miR-15a expression [84], demonstrating a potential molecular mechanism induced by PE to reduce UCP-2 expression and improve insulin secretion.

In this context, miR-96 also participates in the regulation of the insulin secretion process, this miRNA is overexpressed in the serum of patients with T2DM, inhibits the calcium voltage-gated channel subunit $\alpha 1 \mathrm{E}$ (CACNA1E) gene, a voltage-dependent calcium channel [28], impairing insulin secretion. On the other hand, PE reduced the expression of miR-96 [85], suggesting that in this way, PE can increase CACNA1E expression and improve insulin secretion.

MiR-192 is downregulated in the plasma of patients with T2DM [29], promoting increased expression of the glucocorticoid receptor (GCR) that favors insulin resistance, lipid accumulation, lipogenesis and hepatic microvesicular steatosis [118]. In contrast, PE induces overexpression of miR-192 [86], which can minimize GCR expression by attenuating insulin resistance, reducing lipid accumulation and lipogenesis.

Likewise, miR-532 also has reduced expression in patients with T2DM and is associated with insulin resistance [29] and inflammation [119]. Downregulation of miR-532 results in overexpression of your targets, apoptosis signalregulating kinase 1 (ASK1) and pro-apoptotic gene homologous Bcl-2 Antagonist/Killer 1 (BAK1), that induce apop- tosis [120]. ASK1 is an inflammation-derived kinase that activates other kinases as well as p38 mitogen-activated protein kinases (p38 MAPKs) that control several inflammatory [121], apoptotic and fibrotic processes in T2DM [122].

On the other hand, cycle ergometer PE increased the expression of miR-532 [87], which can be an excellent strategy for reducing ASK1, BAK1, and p38 MAPKs, and inducing the inhibition of the inflammatory, fibrotic and apoptotic process in T2DM, in addition, it may be a new beneficial molecular mechanism induced by PE in T2DM and new therapeutic target.

\section{Conclusions}

In this review, it was identified that many miRNAs have their expression pattern modified during and after $\mathrm{PE}$ and sometimes different from the expression pattern in T2DM. These finds suggest that PE beneficially modulates the expression of the miRNAs and signaling pathways of the processes of secretion and insulin resistance, lipogenesis, apoptosis, inflammation and fibrosis, promoting beneficial changes in the clinical parameters of individuals with T2DM.

\section{Abbreviations}

T2DM, Type 2 Diabetes Mellitus; MiRNAs, MicroRNAs; PE, Physical Exercise; IRS-1, insulin receptor substrate-1; PI3K, phosphatidylinositol 3-kinase; PKB, protein kinase B; GLUT4, glucose transporter 4; RNA, ribonucleic acids; mRNA, messenger ribonucleic acids; ATP, adenosine triphosphate; PPAR $\alpha$, peroxisome proliferatoractivated alpha; ROS, reactive oxygen species; NOC2, nucleolar complex protein 2 ; TNF- $\alpha$, tumor necrosis factor- 
$\alpha$; NOS2, nitric oxide synthase-2; IL-12, interleukin-12; TTP, tristetrapolin; IL-6, interleukin-6; IL-18, interleukin18; PGC- $1 \alpha$, peroxisome proliferator-activated receptor gamma coactivator-1 alpha; UCP-2, uncoupling protein2; CACNA1E, calcium voltage-gated channel subunit $\alpha 1$ E; GCR, glucocorticoid receptor; ASK1, apoptosis signalregulating kinase 1; BAK1, pro-apoptotic gene homologous Bcl-2 Antagonist/Killer 1; p38 MAPKs, p38 mitogenactivated protein kinases.

\section{Author contributions}

ACI-C, RALDS and TF wrote the manuscript; ACI$\mathrm{C}$, RALDS and TF performed the literature research; ACIC, RALDS, TF and EMO analyzed and critically discussed the data; ACI-C, LR, BSFS, RAJ, TF and EMO performed formal analysis and supervision; ACI-C, TF and EMO performed review and final editing.

\section{Ethics approval and consent to participate}

Not applicable.

\section{Acknowledgment}

We would like to express our gratitude to all those who helped us during the writing of this manuscript.

\section{Funding}

This study was partially funded by Coordenação de Aperfeiçoamento de Pessoal de Nível Superior - Brasil (CAPES) - Financial Code 001. This article was funded by Fundação Oswaldo Cruz (FIOCRUZ).

\section{Conflict of interest}

The authors declare no conflict of interest.

\section{References}

[1] Association AD. 2. Classification and diagnosis of diabetes: Standards of medical care in diabetesd-2019. Diabetes Care. 2019; 42: S13-S28.

[2] Boudina S, Abel ED. Diabetic cardiomyopathy revisited. Circulation. 2007; 115: 3213-3223.

[3] Petersen MC, Shulman GI. Mechanisms of Insulin Action and Insulin Resistance. Physiological Reviews. 2018; 98: 21332223.

[4] Krook A, Wallberg-Henriksson H, Zierath JR. Sending the signal: molecular mechanisms regulating glucose uptake. Medicine and Science in Sports and Exercise. 2004; 36: 1212-1217.

[5] Jia G, DeMarco VG, Sowers JR. Insulin resistance and hyperinsulinaemia in diabetic cardiomyopathy. Nature Reviews Endocrinology. 2016; 12: 144-153.

[6] Chakraborty C, Doss CGP, Bandyopadhyay S, Agoramoorthy G. Influence of miRNA in insulin signaling pathway and insulin resistance: micro-molecules with a major role in type-2 diabetes. Wiley Interdisciplinary Reviews. 2014; 5: 697-712.

[7] Baek D, Villén J, Shin C, Camargo FD, Gygi SP, Bartel DP. The impact of microRNAs on protein output. Nature. 2008; 455: 6471.

[8] Soci UPR, Fernandes T, Barauna VG, Hashimoto NY, de Fátima Alves Mota G, Rosa KT, et al. Epigenetic control of exer- cise training-induced cardiac hypertrophy by miR-208. Clinical Science. 2016; 130: 2005-2015.

[9] Fernandes T, Magalhães FC, Roque FR, Phillips MI, Oliveira EM. Exercise training prevents the microvascular rarefaction in hypertension balancing angiogenic and apoptotic factors: role of microRNAs-16, -21, and -126. Hypertension. 2012; 59: $513-$ 520.

[10] Improta-Caria AC, Aras R. Treinamento com Exercício Físico e Doença de Chagas: Função Potencial dos MicroRNAs. Arquivos Brasileiros De Cardiologia. 2021; 117: 132-141.

[11] Improta Caria AC, Nonaka CKV, Pereira CS, Soares MBP, Macambira SG, Souza BSDF. Exercise Training-Induced Changes in MicroRNAs: Beneficial Regulatory Effects in Hypertension, Type 2 Diabetes, and Obesity. International Journal of Molecular Sciences. 2018; 19: 1-36.

[12] Temple KA, Tjaden AH, Atkinson KM, Barengolts E, Hannon TS, Mather KJ, et al. Association of habitual daily physical activity with glucose tolerance and B-cell function in adults with impaired glucose tolerance or recently diagnosed type 2 diabetes from the Restoring Insulin Secretion (RISE) study. Diabetes Care. 2019; 42: 1521-1529.

[13] Liu S, Zheng F, Xie K, Xie M, Jiang L, Cai Y. Exercise Reduces Insulin Resistance in Type 2 Diabetes Mellitus via Mediating the lncRNA MALAT1/MicroRNA-382-3p/Resistin Axis. Molecular Therapy - Nucleic Acids. 2019; 18: 34-44.

[14] De Sousa RAL, Improta-Caria AC, Jesus-Silva FMD, Magalhães CODE, Freitas DA, Lacerda ACR, et al. High-intensity resistance training induces changes in cognitive function, but not in locomotor activity or anxious behavior in rats induced to type 2 diabetes. Physiology \& Behavior. 2020; 223: 112998.

[15] Zheng Y, Ley SH, Hu FB. Global aetiology and epidemiology of type 2 diabetes mellitus and its complications. Nature Reviews Endocrinology. 2018; 14: 88-98.

[16] Prentki M. Islet $\beta$ cell failure in type 2 diabetes. Journal of Clinical Investigation. 2006; 116: 1802-1812.

[17] Wang L, Cai Y, Jian L, Cheung CW, Zhang L, Xia Z. Impact of peroxisome proliferator-activated receptor- $\alpha$ on diabetic cardiomyopathy. Cardiovascular Diabetology. 2021; 20: 1-15.

[18] Harmancey R, Haight DL, Watts KA, Taegtmeyer H. Chronic Hyperinsulinemia Causes Selective Insulin Resistance and down-regulates Uncoupling Protein 3 (UCP3) through the Activation of Sterol Regulatory Element-binding Protein (SREBP)-1 Transcription Factor in the Mouse Heart. The Journal of Biological Chemistry. 2015; 290: 30947-30961.

[19] Finck BN, Lehman JJ, Leone TC, Welch MJ, Bennett MJ, Kovacs A, et al. The cardiac phenotype induced by PPARalpha overexpression mimics that caused by diabetes mellitus. The Journal of Clinical Investigation. 2002; 109: 121-130.

[20] Kyriazis ID, Hoffman M, Gaignebet L, Lucchese AM, Markopoulou E, Palioura D, et al. KLF5 is Induced by FOXO1 and Causes Oxidative Stress and Diabetic Cardiomyopathy. Circulation Research. 2021; 128: 335-357.

[21] Thai MV, Guruswamy S, Cao KT, Pessin JE, Olson AL. Myocyte enhancer factor 2 (MEF2)-binding site is required for GLUT4 gene expression in transgenic mice. Regulation of MEF2 DNA binding activity in insulin-deficient diabetes. The Journal of Biological Chemistry. 1998; 273: 14285-14292.

[22] Frati G, Schirone L, Chimenti I, Yee D, Biondi-Zoccai G, Volpe $\mathrm{M}$, et al. An overview of the inflammatory signalling mechanisms in the myocardium underlying the development of diabetic cardiomyopathy. Cardiovascular Research. 2017; 113: 378-388.

[23] De Rosa S, Arcidiacono B, Chiefari E, Brunetti A, Indolfi C, Foti DP. Type 2 diabetes mellitus and cardiovascular disease: Genetic and epigenetic links. Frontiers in Endocrinology. 2018; 9: $1-13$. 
[24] Huntzinger E, Izaurralde E. Gene silencing by microRNAs: contributions of translational repression and mRNA decay. Nature Reviews Genetics. 2011; 12: 99-110.

[25] Baldeón R L, Weigelt K, de Wit H, Ozcan B, van Oudenaren A, Sempértegui F, et al. Decreased serum level of miR-146a as sign of chronic inflammation in type 2 diabetic patients. PLoS ONE. 2014; 9: e115209.

[26] Karolina DS, Armugam A, Tavintharan S, Wong MTK, Lim SC, Sum CF, et al. MicroRNA 144 impairs insulin signaling by inhibiting the expression of insulin receptor substrate 1 in type 2 diabetes mellitus. PLoS ONE. 2011; 6: e22839.

[27] Jiménez-Lucena R, Camargo A, Alcalá-Diaz JF, RomeroBaldonado C, Luque RM, van Ommen B, et al. A plasma circulating miRNAs profile predicts type 2 diabetes mellitus and prediabetes: from the Cordioprev study. Experimental \& Molecular Medicine. 2018; 50: 1-12.

[28] Yang Z, Chen L, Hong M, Chen Y, Yang X, Tang S, et al. Serum microRNA profiling and bioinformatics analysis of patients with type 2 diabetes mellitus in a Chinese population. Molecular Medicine Reports. 2017; 15: 2143-2153.

[29] Ortega FJ, Mercader JM, Moreno-Navarrete JM, Rovira O, Guerra E, Esteve E, et al. Profiling of circulating microRNAs reveals common microRNAs linked to type 2 diabetes that change with insulin sensitization. Diabetes Care. 2014; 37: 1375-1383.

[30] Jiao Y, Zhu M, Mao X, Long M, Du X, Wu Y, et al. MicroRNA130a expression is decreased in Xinjiang Uygur patients with type 2 diabetes mellitus. American Journal of Translational Research. 2015; 7: 1984-1991.

[31] Prabu P, Rome S, Sathishkumar C, Aravind S, Mahalingam B, Shanthirani CS, et al. Circulating MiRNAs of 'Asian Indian Phenotype' Identified in Subjects with Impaired Glucose Tolerance and Patients with Type 2 Diabetes. PLoS ONE. 2015; 10: e0128372.

[32] Yan S, Wang T, Huang S, Di Y, Huang Y, Liu X, et al. Differential expression of microRNAs in plasma of patients with prediabetes and newly diagnosed type 2 diabetes. Acta Diabetologica. 2016; 53: 693-702.

[33] Kong L, Zhu J, Han W, Jiang X, Xu M, Zhao Y, et al. Significance of serum microRNAs in pre-diabetes and newly diagnosed type 2 diabetes: a clinical study. Acta Diabetologica. 2011; 48: 61-69.

[34] Brennan E, Wang B, McClelland A, Mohan M, Marai M, Beuscart O, et al. Protective Effect of let-7 miRNA Family in Regulating Inflammation in Diabetes-Associated Atherosclerosis. Diabetes. 2017; 66: 2266-2277.

[35] Pescador N, Pérez-Barba M, Ibarra JM, Corbatón A, MartínezLarrad MT, Serrano-Ríos M. Serum circulating microRNA profiling for identification of potential type 2 diabetes and obesity biomarkers. PLoS ONE. 2013; 8: e77251.

[36] Higuchi C, Nakatsuka A, Eguchi J, Teshigawara S, Kanzaki M, Katayama A, et al. Identification of circulating miR-101, miR375 and miR- 802 as biomarkers for type 2 diabetes. Metabolism. 2015; 64: 489-497.

[37] Yang Z, Chen H, Si H, Li X, Ding X, Sheng Q, et al. Serum miR23a, a potential biomarker for diagnosis of pre-diabetes and type 2 diabetes. Acta Diabetologica. 2014; 51: 823-831.

[38] de Gonzalo-Calvo D, van der Meer RW, Rijzewijk LJ, Smit JWA, Revuelta-Lopez E, Nasarre L, et al. Serum microRNA-1 and microRNA-133a levels reflect myocardial steatosis in uncomplicated type 2 diabetes. Scientific Reports. 2017; 7: 47.

[39] Zampetaki A, Kiechl S, Drozdov I, Willeit P, Mayr U, Prokopi $\mathrm{M}$, et al. Plasma microRNA profiling reveals loss of endothelial miR-126 and other microRNAs in type 2 diabetes. Circulation Research. 2010; 107: 810-817.

[40] Zhang T, Li L, Shang Q, Lv C, Wang C, Su B. Circulating miR126 is a potential biomarker to predict the onset of type 2 dia- betes mellitus in susceptible individuals. Biochemical and Biophysical Research Communications. 2015; 463: 60-63.

[41] Liu Y, Gao G, Yang C, Zhou K, Shen B, Liang H, et al. The role of circulating microRNA-126 (miR-126): a novel biomarker for screening prediabetes and newly diagnosed type 2 diabetes mellitus. International Journal of Molecular Sciences. 2014; 15: 10567-10577.

[42] Olivieri F, Spazzafumo L, Bonafè M, Recchioni R, Prattichizzo F, Marcheselli F, et al. MiR-21-5p and miR-126a-3p levels in plasma and circulating angiogenic cells: relationship with type 2 diabetes complications. Oncotarget. 2015; 6: 35372-35382.

[43] Sun L, Li X, Li G, Dai B, Tan W. Actinidia chinensis Planch. Improves the Indices of Antioxidant and Anti-Inflammation Status of Type 2 Diabetes Mellitus by Activating Keap1 and Nrf2 via the Upregulation of MicroRNA-424. Oxidative Medicine and Cellular Longevity. 2017; 2017: 7038789.

[44] Nunez Lopez YO, Garufi G, Seyhan AA. Altered levels of circulating cytokines and microRNAs in lean and obese individuals with prediabetes and type 2 diabetes. Molecular BioSystems. 2017; 13: 106-121.

[45] Seyhan AA, Nunez Lopez YO, Xie H, Yi F, Mathews C, Pasarica $\mathrm{M}$, et al. Pancreas-enriched miRNAs are altered in the circulation of subjects with diabetes: a pilot cross-sectional study. Scientific Reports. 2016; 6: 31479.

[46] Nigi L, Grieco GE, Ventriglia G, Brusco N, Mancarella F, Formichi C, et al. MicroRNAs as Regulators of Insulin Signaling: Research Updates and Potential Therapeutic Perspectives in Type 2 Diabetes. International Journal of Molecular Sciences. 2018; 19.

[47] Poy MN, Eliasson L, Krutzfeldt J, Kuwajima S, Ma X, Macdonald PE, et al. A pancreatic islet-specific microRNA regulates insulin secretion. Nature. 2004; 432: 226-230.

[48] Poy MN, Hausser J, Trajkovski M, Braun M, Collins S, Rorsman P, et al. MiR-375 maintains normal pancreatic $\alpha$ - and $\beta$-cell mass. Proceedings of the National Academy of Sciences. 2009; 106: 5813-5818.

[49] Lovis P, Gattesco S, Regazzi R. Regulation of the expression of components of the exocytotic machinery of insulin-secreting cells by microRNAs. Biological Chemistry. 2008; 389: 305312.

[50] Joglekar MV, Parekh VS, Mehta S, Bhonde RR, Hardikar AA. MicroRNA profiling of developing and regenerating pancreas reveal post-transcriptional regulation of neurogenin3. Developmental Biology. 2007; 311: 603-612.

[51] Correa-Medina M, Bravo-Egana V, Rosero S, Ricordi C, Edlund $\mathrm{H}$, Diez J, et al. MicroRNA miR-7 is preferentially expressed in endocrine cells of the developing and adult human pancreas. Gene Expression Patterns. 2009; 9: 193-199.

[52] Wan S, Wang J, Wang J, Wu J, Song J, Zhang C, et al. Increased serum miR-7 is a promising biomarker for type 2 diabetes mellitus and its microvascular complications. Diabetes Research and Clinical Practice. 2017; 130: 171-179.

[53] Fernández-de Frutos M, Galán-Chilet I, Goedeke L, Kim B, Pardo-Marqués V, Pérez-García A, et al. MicroRNA 7 Impairs Insulin Signaling and Regulates $A \beta$ Levels through Posttranscriptional Regulation of the Insulin Receptor. Molecular and Cellular Biology. 2019; 39: e00170-19.

[54] Shantikumar S, Caporali A, Emanueli C. Role of microRNAs in diabetes and its cardiovascular complications. Cardiovascular Research. 2012; 93: 583-593.

[55] Park S, Lee JH, Ha M, Nam J, Kim VN. MiR-29 miRNAs activate 53 by targeting $\mathrm{p} 85 \alpha$ and $\mathrm{CDC} 42$. Nature Structural \& Molecular Biology. 2009; 16: 23-29.

[56] Ling H, Ou H, Feng S, Zhang X, Tuo Q, Chen L, et al. Changes in microRNA (miR) Profile and Effects of miR-320 in InsulinResistant 3T3-L1 Adipocytes. Clinical and Experimental Phar- 
macology and Physiology. 2009; 36: e32-e39.

[57] Guo C, Sah JF, Beard L, Willson JKV, Markowitz SD, Guda K. The noncoding RNA, miR-126, suppresses the growth of neoplastic cells by targeting phosphatidylinositol 3-kinase signaling and is frequently lost in colon cancers. Genes Chromosomes \& Cancer. 2008; 47: 939-946.

[58] He A, Zhu L, Gupta N, Chang Y, Fang F. Overexpression of micro ribonucleic acid 29, highly up-regulated in diabetic rats, leads to insulin resistance in 3T3-L1 adipocytes. Molecular Endocrinology. 2007; 21: 2785-2794.

[59] Dávalos A, Goedeke L, Smibert P, Ramírez CM, Warrier NP, Andreo $\mathrm{U}$, et al. MiR-33a/b contribute to the regulation of fatty acid metabolism and insulin signaling. Proceedings of the National Academy of Sciences of the United States of America. 2011; 108: 9232-9237.

[60] Xu J, Liao X, Lu N, Liu W, Wong C. Chromatin-modifying drugs induce miRNA-153 expression to suppress Irs-2 in glioblastoma cell lines. International Journal of Cancer. 2011; 129: 2527-2531.

[61] Esteves JV, Enguita FJ, Machado UF. MicroRNAs-Mediated Regulation of Skeletal Muscle GLUT4 Expression and Translocation in Insulin Resistance. Journal of Diabetes Research. 2017; 2017: 7267910.

[62] Xu R, Zeng G, Wang S, Tao H, Ren L, Zhang Z, et al. Periodontitis promotes the diabetic development of obese rat via miR-147 induced classical macrophage activation. Biomedicine \& Pharmacotherapy. 2016; 83: 892-897.

[63] De Sousa RAL, Harmer AR, Freitas DA, Mendonça VA, Lacerda ACR, Leite HR. An update on potential links between type 2 diabetes mellitus and Alzheimer's disease. Molecular Biology Reports. 2020; 47: 6347-6356.

[64] Improta-Caria AC, Nonaka CKV, Cavalcante BRR, De Sousa RAL, Júnior RA, Souza BS de F. Modulation of microRNAs as a potential molecular mechanism involved in the beneficial actions of physical exercise in Alzheimer disease. International Journal of Molecular Sciences. 2020; 21: 1-35.

[65] Guo J, Li J, Zhao J, Yang S, Wang L, Cheng G, et al. MiRNA$29 \mathrm{c}$ regulates the expression of inflammatory cytokines in diabetic nephropathy by targeting tristetraprolin. Scientific Reports. 2017; 7: 2314.

[66] Bradburn S, Sarginson J, Murgatroyd CA. Association of Peripheral Interleukin-6 with Global Cognitive Decline in Nondemented Adults: a Meta-Analysis of Prospective Studies. Frontiers in Aging Neuroscience. 2018; 9: 438.

[67] Ng A, Tam WW, Zhang MW, Ho CS, Husain SF, McIntyre RS, et al. IL-1 $\beta$, IL-6, TNF- $\alpha$ and CRP in Elderly Patients with Depression or Alzheimer's disease: Systematic Review and MetaAnalysis. Scientific Reports. 2018; 8: 12050.

[68] Arnold SE, Arvanitakis Z, Macauley-Rambach SL, Koenig AM, Wang H, Ahima RS, et al. Brain insulin resistance in type 2 diabetes and Alzheimer disease: concepts and conundrums HHS Public Access. Nature Reviews Neurology. 2018; 14: 168-181.

[69] Colberg SR, Grieco CR. Exercise in the Treatment and Prevention of Diabetes. Current Sports Medicine Reports. 2009; 8: 169-175.

[70] Improta-Caria AC, Soci ÚPR, Pinho CS, Júnior RA, de Sousa RAL, Bessa TCB. Physical exercise and immune system: Perspectives on the COVID-19 pandemic. Revista da Associacao Medica Brasileira. 2021; 67: 102-107.

[71] Khadir A, Kavalakatt S, Cherian P, Warsame S, Abubaker JA, Dehbi M, et al. Physical exercise enhanced heat shock protein 60 expression and attenuated inflammation in the adipose tissue of human diabetic obese. Frontiers in Endocrinology. 2018; 9: 6.

[72] De Sousa RAL, Rodrigues CM, Mendes BF, Improta-Caria AC, Peixoto MFD, Cassilhas RC. Physical exercise protocols in animal models of Alzheimer's disease: a systematic review. Metabolic Brain Disease. 2020; 36: 85-95.

[73] DiMenna FJ, Arad AD. Exercise as 'precision medicine' for insulin resistance and its progression to type 2 diabetes: a research review. BMC Sports Science, Medicine \& Rehabilitation. 2018; 10: 21 .

[74] Augusto R, Sousa L De. Breve Relato da Resistência à Insulina e os Benefícios do Exercício Resistido Intenso no Diabetes Tipo 2. Saúde e Pesquisa. 2014; 7.

[75] De Sousa RAL. Brief report of the effects of the aerobic, resistance, and high-intensity interval training in type 2 diabetes mellitus individuals. International Journal of Diabetes in Developing Countries. 2018; 38: 138-145.

[76] Garber CE, Blissmer B, Deschenes MR, Franklin BA, Lamonte MJ, Lee I, et al. Quantity and Quality of Exercise for Developing and Maintaining Cardiorespiratory, Musculoskeletal, and Neuromotor Fitness in Apparently Healthy Adults: Guidance for prescribing exercise. Medicine \& Science in Sports \& Exercise. 2011; 43: 1334-1359.

[77] Bellavere F, Cacciatori V, Bacchi E, Gemma ML, Raimondo D, Negri $\mathrm{C}$, et al. Effects of aerobic or resistance exercise training on cardiovascular autonomic function of subjects with type 2 diabetes: a pilot study. Nutrition Metabolism and Cardiovascular Diseases. 2018; 28: 226-233.

[78] van Dijk J, Manders RJF, Tummers K, Bonomi AG, Stehouwer CDA, Hartgens F, et al. Both resistance- and endurance-type exercise reduce the prevalence of hyperglycaemia in individuals with impaired glucose tolerance and in insulin-treated and noninsulin-treated type 2 diabetic patients. Diabetologia. 2012; 55: $1273-1282$.

[79] Liang H, Ward WF. PGC- $1 \alpha$ : a key regulator of energy metabolism. Advances in Physiology Education. 2006; 30: 145151.

[80] Boström P, Wu J, Jedrychowski MP, Korde A, Ye L, Lo JC, et al. A PGC1- $\alpha$-dependent myokine that drives brown-fat-like development of white fat and thermogenesis. Nature. 2012; 481: 463-468.

[81] Lourenco MV, Frozza RL, de Freitas GB, Zhang H, Kincheski GC, Ribeiro FC, et al. Exercise-linked FNDC5/irisin rescues synaptic plasticity and memory defects in Alzheimer's models. Nature Medicine. 2019; 25: 165-175.

[82] De Sousa RAL, Improta-Caria AC, Souza BS de F. ExerciseLinked Irisin: Consequences on Mental and Cardiovascular Health in Type 2 Diabetes. International Journal of Molecular Sciences. 2021; 22: 2199.

[83] Nielsen S, Åkerström T, Rinnov A, Yfanti C, Scheele C, Pedersen BK, et al. The miRNA plasma signature in response to acute aerobic exercise and endurance training. PLoS ONE. 2014; 9: e87308.

[84] Radom-Aizik S, Zaldivar F, Leu S, Adams GR, Oliver S, Cooper DM. Effects of exercise on microRNA expression in young males peripheral blood mononuclear cells. Clinical and Translational Science. 2012; 5: 32-38.

[85] Radom-Aizik S, Zaldivar F, Oliver S, Galassetti P, Cooper DM. Evidence for microRNA involvement in exercise-associated neutrophil gene expression changes. Journal of Applied Physiology. 2010; 109: 252-261.

[86] Radom-Aizik S, Zaldivar F, Haddad F, Cooper DM. Impact of brief exercise on peripheral blood NK cell gene and microRNA expression in young adults. Journal of Applied Physiology. 2013; 114: 628-636.

[87] Radom-Aizik S, Zaldivar FP, Haddad F, Cooper DM. Impact of brief exercise on circulating monocyte gene and microRNA expression: implications for atherosclerotic vascular disease. Brain Behavior and Immunity. 2014; 39: 121-129.

[88] Baggish AL, Hale A, Weiner RB, Lewis GD, Systrom D, Wang 
$\mathrm{F}$, et al. Dynamic regulation of circulating microRNA during acute exhaustive exercise and sustained aerobic exercise training. The Journal of Physiology. 2011; 589: 3983-3994.

[89] Sawada S, Kon M, Wada S, Ushida T, Suzuki K, Akimoto T. Profiling of Circulating MicroRNAs after a Bout of Acute Resistance Exercise in Humans. PLoS ONE. 2013; 8: e70823.

[90] Aoi W, Ichikawa H, Mune K, Tanimura Y, Mizushima K, Naito $\mathrm{Y}$, et al. Muscle-enriched microRNA miR-486 decreases in circulation in response to exercise in young men. Frontiers in Physiology. 2013; 4: 80

[91] Banzet S, Chennaoui M, Girard O, Racinais S, Drogou C, Chalabi $\mathrm{H}$, et al. Changes in circulating microRNAs levels with exercise modality. Journal of Applied Physiology. 2013; 115: $1237-$ 1244.

[92] Uhlemann M, Möbius-Winkler S, Fikenzer S, Adam J, Redlich M, Möhlenkamp S, et al. Circulating microRNA-126 increases after different forms of endurance exercise in healthy adults. European Journal of Preventive Cardiology. 2014; 21: 484-491.

[93] Baggish AL, Park J, Min P, Isaacs S, Parker BA, Thompson PD, et al. Rapid upregulation and clearance of distinct circulating microRNAs after prolonged aerobic exercise. Journal of Applied Physiology. 2014; 116: 522-531.

[94] Mooren FC, Viereck J, Krüger K, Thum T. Circulating microRNAs as potential biomarkers of aerobic exercise capacity. American Journal of Physiology. Heart and Circulatory Physiology. 2014; 306: H557-H563.

[95] Gomes CPC, Oliveira-Jr GP, Madrid B, Almeida JA, Franco OL, Pereira RW. Circulating miR-1, miR-133a, and miR-206 levels are increased after a half-maraathon run. Biomarkers. 2014; 19: 585-589.

[96] Zhang T, Birbrair A, Wang Z, Messi ML, Marsh AP, Leng I, et al. Improved knee extensor strength with resistance training associates with muscle specific miRNAs in older adults. Experimental Gerontology. 2015; 62: 7-13.

[97] Dias RG, Silva MSM, Duarte NE, Bolani W, Alves CR, Junior JRL, et al. PBMCs express a transcriptome signature predictor of oxygen uptake responsiveness to endurance exercise training in men. Physiological Genomics. 2015; 47: 13-23.

[98] Liu X, Xiao J, Zhu H, Wei X, Platt C, Damilano F, et al. MiR-222 is Necessary for Exercise-Induced Cardiac Growth and Protects against Pathological Cardiac Remodeling. Cell Metabolism. 2015; 21: 584-595.

[99] de Gonzalo-Calvo D, Dávalos A, Montero A, García-González Á, Tyshkovska I, González-Medina A, et al. Circulating inflammatory miRNA signature in response to different doses of aerobic exercise. Journal of Applied Physiology. 2015; 119: 124134.

[100] Clauss S, Wakili R, Hildebrand B, Kääb S, Hoster E, Klier I, et al. MicroRNAs as Biomarkers for Acute Atrial Remodeling in Marathon Runners (the miRathon Study-a Sub-Study of the Munich Marathon Study). PLoS ONE. 2016; 11: e0148599.

[101] Min P, Park J, Isaacs S, Taylor BA, Thompson PD, Troyanos C, et al. Influence of statins on distinct circulating microRNAs during prolonged aerobic exercise. Journal of Applied Physiology. 2016; 120: 711-720.

[102] Cui SF, Wang C, Yin X, Tian D, Lu QJ, Zhang CY, et al. Similar Responses of Circulating MicroRNAs to Acute High-Intensity Interval Exercise and Vigorous-Intensity Continuous Exercise. Frontiers in Physiology. 2016; 7: 102.

[103] Denham J, Prestes PR. Muscle-Enriched MicroRNAs Isolated from Whole Blood Are Regulated by Exercise and Are Potential Biomarkers of Cardiorespiratory Fitness. Frontiers in Genetics. 2016; 7: 196.

[104] Zhang T, Brinkley TE, Liu K, Feng X, Marsh AP, Kritchevsky $\mathrm{S}$, et al. Circulating MiRNAs as biomarkers of gait speed responses to aerobic exercise training in obese older adults. Aging.
2017; 9: 900-913.

[105] Horak M, Zlamal F, Iliev R, Kucera J, Cacek J, Svobodova L, et al. Exercise-induced circulating microRNA changes in athletes in various training scenarios. PLoS ONE. 2018; 13: e0191060.

[106] Li Y, Yao M, Zhou Q, Cheng Y, Che L, Xu J, et al. Dynamic regulation of circulating microRNAs during acute exercise and long-term exercise training in basketball athletes. Frontiers in Physiology. 2018; 9.

[107] de Gonzalo-Calvo D, Dávalos A, Fernández-Sanjurjo M, Amado-Rodríguez L, Díaz-Coto S, Tomás-Zapico C, et al. Circulating microRNAs as emerging cardiac biomarkers responsive to acute exercise. International Journal of Cardiology. 2018; 264: $130-136$.

[108] Westerberg E, Molin CJ, Lindblad I, Emtner M, Punga AR. Physical exercise in myasthenia gravis is safe and improves neuromuscular parameters and physical performance-based measures: a pilot study. Muscle \& Nerve. 2017; 56: 207-214.

[109] Margolis LM, Berryman CE, Murphy NE, Carrigan CT, Young AJ, Carbone JW, et al. PI3K-AKT-FOXO1 pathway targeted by skeletal muscle microRNA to suppress proteolytic gene expression in response to carbohydrate intake during aerobic exercise. Physiological Reports. 2018; 6: e13931.

[110] Zhou Q, Shi C, Lv Y, Zhao C, Jiao Z, Wang T. Circulating microRNAs in Response to Exercise Training in Healthy Adults. Frontiers in Genetics. 2020; 11.

[111] Olioso D, Dauriz M, Bacchi E, Negri C, Santi L, Bonora E, et al. Effects of Aerobic and Resistance Training on Circulating Micro-RNA Expression Profile in Subjects with Type 2 Diabetes. The Journal of Clinical Endocrinology \& Metabolism. 2019; 104: 1119-1130.

[112] Simaitis S, Schulte-Körne B, Schiffer T, Bloch W, Predel HG, Brixius $\mathrm{K}$, et al. Evidence for Training-Induced Changes in miRNA Levels in the Skeletal Muscle of Patients With Type 2 Diabetes Mellitus. Frontiers in Physiology. 2020; 11: 1554.

[113] Souza RWA, Fernandez GJ, Cunha JPQ, Piedade WP, Soares LC, Souza PAT, et al. Regulation of cardiac microRNAs induced by aerobic exercise training during heart failure. American Journal of Physiology-Heart and Circulatory Physiology. 2015; 309: H1629-H1641.

[114] Karbiener M, Fischer C, Nowitsch S, Opriessnig P, Papak C, Ailhaud G, et al. MicroRNA miR-27b impairs human adipocyte differentiation and targets PPAR $\gamma$. Biochemical and Biophysical Research Communications. 2009; 390: 247-251.

[115] Yu Y, Du H, Wei S, Feng L, Li J, Yao F, et al. AdipocyteDerived Exosomal MiR-27a Induces Insulin Resistance in Skeletal Muscle through Repression of PPAR $\gamma$. Theranostics. 2018; 8: 2171-2188.

[116] Sun L, Jiang B, Li W, Zou J, Shi Y, Liu Z. MicroRNA-15a positively regulates insulin synthesis by inhibiting uncoupling protein-2 expression. Diabetes Research and Clinical Practice. 2011; 91: 94-100.

[117] Chan CB, MacDonald PE, Saleh MC, Johns DC, Marbàn E, Wheeler MB. Overexpression of uncoupling protein 2 inhibits glucose-stimulated insulin secretion from rat islets. Diabetes. 1999; 48: 1482-1486.

[118] Wang Z, Miu K, Zhang X, Wan AT, Lu G, Cheung H, et al. Hepatic miR-192-3p reactivation alleviates steatosis by targeting glucocorticoid receptor. JHEP Reports. 2020; 2: 100179.

[119] Esteves JV, Yonamine CY, Pinto-Junior DC, GerlingerRomero F, Enguita FJ, Machado UF. Diabetes Modulates MicroRNAs 29b-3p, 29c-3p, 199a-5p and 532-3p Expression in Muscle: Possible Role in GLUT4 and HK2 Repression. Frontiers in Endocrinology. 2018; 9: 536.

[120] Chen F, Shen Y, Liu Y, Wang H, Liang C, Luo M. Inflammation-dependent downregulation of miR-532-3p mediates apoptotic signaling in human sarcopenia through targeting 
BAK1. International Journal of Biological Sciences. 2020; 16: 1481-1494.

[121] Dinesh P, Kalaiselvan S, Sujitha S, Rasool M. MicroRNA-5323p Regulates Pro-Inflammatory Human THP-1 Macrophages by Targeting ASK1/p38 MAPK Pathway. Inflammation. 2020; 44: 229-242.
[122] Yamamoto E, Dong Y, Kataoka K, Yamashita T, Tokutomi Y, Matsuba S, et al. Olmesartan prevents cardiovascular injury and hepatic steatosis in obesity and diabetes, accompanied by apoptosis signal regulating kinase-1 inhibition. Hypertension. 2008; 52: $573-580$. 


\section{Temporary page!}

${ }_{\mathrm{HT}} \mathrm{T}$ was unable to guess the total number of pages correctly. As there was some unprocessed data that should have been added to the final page this extra page has been added to receive it.

If you rerun the document (without altering it) this surplus page will go away, because ${ }_{\mathrm{AT}} \mathrm{T} \mathrm{X}$ now knows how many pages to expect for this document. 